\title{
La place de la modélisation dans l'enseignement de l'algèbre élémentaire : pratiques institutionnelles et pratiques enseignantes dans le système éducatif tunisien
}

\section{The place of modelling in the teaching of elementary algebra: institutional practices and teaching practices in the Tunisian education system}

\author{
Sonia Ben Nejma ${ }^{1 *}$ \\ ${ }^{1}$ Université de Carthage, Laboratoire LaRINA, Faculté des sciences de Bizerte, Tunisie
}

\begin{abstract}
Résumé. Considérant l'évolution du curriculum d'enseignement des mathématiques vers une approche de la discipline par la modélisation, nous nous intéressons ici, aux contraintes de l'implantation de cette démarche dans l'enseignement secondaire tunisien. Cette question est abordée à travers deux dimensions des pratiques enseignantes, une dimension institutionnelle qui se base sur une analyse des praxéologies mathématiques développées par l'institution autour de la modélisation, notamment, dans le domaine de l'algèbre élémentaire, au cycle secondaire, et une dimension professionnelle qui est approchée par les pratiques déclaratives des enseignants, selon les points de vue, épistémologique, fonctionnel et organisationnel. Les analyses sont conduites dans le cadre de la Théorie Anthropologique du Didactique et s'inspirent du modèle proposé par Blum et Leiss. L'articulation de ces deux dimensions des pratiques permet de mieux comprendre la logique d'action des enseignants et les obstacles rencontrés dans la mise en œuvre de cette démarche au niveau des pratiques effectives.
\end{abstract}

\begin{abstract}
Considering the evolution of the curriculum of teaching mathematics towards an approach to the discipline through modeling, we are interested here, the constraints of the implementation of this approach in Tunisian secondary school. This question is addressed through two dimensions of teaching practices, an institutional dimension which is based on an analysis of the mathematical praxeologies developed by the institution around modeling, in particular, in the field of elementary algebra, at the secondary level, and a professional dimension that is approached by the declarative practices of teachers, according to the viewpoints,
\end{abstract}

*Corresponding author: sonianejma@yahoo.com, sonia.bennejma@fssb.u-carthage.tn 
epistemological, functional and organizational. The analyses are conducted within the framework of the Anthropological Theory of Didactics and are based on the model proposed by Blum and Leiss. The articulation of these two dimensions of practice makes it possible to better understand the logic of action of teachers and the obstacles encountered in the implementation of this approach at the level of effective practices.

\section{Introduction et problématique}

Notre réflexion sur l'enseignement des mathématiques par la modélisation est motivée par plusieurs constats. D'abord, les courants didactiques internationaux assez influents ces dernières années en didactique des disciplines (Realistic Mathematics Education, l'Anchored instruction...) et les travaux engagés par des chercheurs internationaux $[1,2,3,4]$ placent la résolution de problèmes au centre des apprentissages en mathématiques. Celle-ci semble influencée par deux écoles. L'école vygotskienne qui considère que la résolution de problèmes permet de développer des compétences plus pratiques dans une perspective socioconstructiviste. Ce n'est plus seulement par ce que l'enseignant transmet, et par les formes de mise en activité des élèves confrontés à des situations problèmes, que les élèves apprennent, c'est par des mises en interactivité (entre élèves et entre enseignant et élèves) que le savoir se construit. Il s'agit plus d'une modalité pédagogique, on apprend par la résolution d'activités de toutes sortes. L'école piagétienne, pour sa part, considère que le problème devient le moyen privilégié pour donner du sens aux connaissances enseignées. Ces travaux contribuent à éclairer les différents aspects liés à cette pratique mais aussi les enjeux cognitifs et épistémologiques impliqués dans ce processus, notamment lorsque la modélisation intervient comme un objet d'apprentissage. Ces recherches permettent, entre autres de dégager les multiples fonctions de la modélisation dans la résolution de problèmes, l'application des connaissances, la construction de nouveaux apprentissages et le développement des démarches d'investigation et des compétences interdisciplinaires. Cependant, dans le contexte scolaire, l'enseignement et l'apprentissage des modèles et de la modélisation posent de grands défis qu'il est intéressant d'explorer. Ensuite, les orientations pédagogiques et didactiques et les changements introduits dans les curricula de mathématiques au secondaire, dans de nombreux pays dont la Tunisie, résident dans l'orientation pragmatique de l'implantation d'un curriculum d'enseignement centré sur la modélisation de situations extra-mathématiques en rapport avec une grande variété de domaines d'expérience. Finalement, les constats établis par les rapports de l'OCDE (2019) concernent les faibles performances des élèves obtenus en général dans les items consacrés à la résolution de problèmes concrets ou extra-mathématiques dans le programme international pour le suivi des acquis des élèves. Les nouveaux cadres épistémologiques de PISA placent ce processus au centre des évaluations des apprentissages en mathématiques et soulignent l'importance d'évaluer les compétences en mathématiques des élèves de 15-16 ans à formuler, employer et interpréter les mathématiques dans de multiples contextes, pour décrire, expliquer et prévoir des phénomènes, afin de mieux appréhender les liens entre mathématique et réalité. L'indicateur utilisé est le score moyen, un bon élève en mathématiques, selon PISA, est sensé comprendre le rôle qu'elles jouent dans le monde, en vue de se comporter en citoyen constructif, engagé et réfléchi, c'est-à-dire poser des jugements et prendre des décisions en toute connaissance de cause. Dans le contexte tunisien, le curriculum d'enseignement implanté dans le cycle secondaire montre une volonté délibérée des concepteurs des programmes à vouloir intégrer simultanément la pratique de la modélisation en mathématiques avec l'introduction de nouveaux objets de savoir ou la construction de nouvelles techniques. Dès le premier cycle, et bien avant avec les problèmes 
d'arithmétique au primaire, les élèves sont conviés à acquérir une diversité de modèles qui font partie des contenus prescrits. D'abord, les modèles sont considérés comme des outils qui permettent de faire une description qualitative des phénomènes, par la suite, ils ont pour fonction de les décrire quantitativement, rarement de manière qualitative. Cette démarche fait désormais partie des pratiques scolaires à caractère scientifique et dans une perspective disciplinaire. Ce qui amène à s'interroger sur les contraintes de la mise en œuvre d'une telle approche sur le terrain et des défis qu'elle pose. Les recherches que nous avons menées dans le contexte tunisien autour des équations algébriques [5-7] ont mis en avant une résistance des pratiques enseignantes aux changements induits par la nouvelle réforme. Les résultats obtenus pointent des difficultés d'adaptation et les études de cas menées laissent entrevoir des pratiques apprêtées souvent décalées par rapport aux pratiques institutionnelles attendues, même si les situations proposées par ces enseignants étaient choisies du manuel officiel. Nous nous appuyons sur les résultats de ces travaux pour explorer, du côté des pratiques déclaratives des enseignants, les raisons qui peuvent expliquer la robustesse des pratiques d'enseignement autour de la modélisation au regard des changements apportés par le système institutionnel tunisien. Il s'agit pour nous de fonder une réflexion didactique sur l'écart observé entre le curriculum implanté et le curriculum prescrit sous l'angle de la visibilité pour les enseignants des enjeux épistémologiques sous-jacents à cette réforme.

Nous nous centrons plus particulièrement sur le domaine de l'algèbre élémentaire à la transition au cycle secondaire. Il s'agit, en effet, d'un moment charnière entre deux cycles d'enseignement, l'enseignement de base et le cycle secondaire qui s'accompagne d'une évolution des apprentissages algébriques et d'un développement des aspects fonctionnels (variable, dépendance fonctionnelle, variation, graphique). Cette transition s'accompagne entre-autres d'un changement de langue ${ }^{\dagger}$ dont l'impact sur les pratiques de modélisation fait l'objet des travaux que nous avons conduit dans ce contexte [8-10]. Dans cet article, nous cherchons à mettre au jour des raisons explicatives de la stabilité des pratiques enseignantes qui semblent centrées sur les techniques algébriques au détriment du développement de la démarche de modélisation. Comment les pratiques enseignantes prennent-elles en compte les injonctions institutionnelles au regard de la modélisation et de l'interdisciplinarité ?

Deux dimensions d'analyse des pratiques enseignantes sont considérées dans cette étude, une dimension institutionnelle qui s'appuie sur une analyse du programme et du manuel officiel relatif à la première année du secondaire tunisien et une dimension professionnelle abordée par une analyse des pratiques déclarées d'enseignants de mathématiques concernés par ce niveau d'enseignement.

\section{Cadre théorique}

\subsection{Rapport institutionnel/Rapport personnel}

Ce travail s'inscrit dans le cadre de la théorie anthropologique de la didactique [11] qui permet de caractériser un système de contraintes qui pèse sur les pratiques enseignantes, sous l'angle de la dialectique des rapports institutionnels et personnels des enseignants à la démarche de modélisation dans l'institution première année du secondaire. Le rapport personnel d'un individu $\mathrm{x}$ à un objet $\mathrm{O}$, expression par laquelle on désigne le système, noté $\mathrm{R}(\mathrm{x} ; \mathrm{O})$, de toutes les interactions que $\mathrm{x}$ peut avoir avec l'objet $\mathrm{O}$ que $\mathrm{x}$ le manipule, l'utilise, en parle, en rêve, etc. On dira que $\mathrm{O}$ existe pour $\mathrm{x}$ si le rapport personnel de $\mathrm{x}$ à $\mathrm{O}$ est « non

\footnotetext{
${ }^{\dagger}$ Le contexte tunisien est bilingue et son système éducatif se caractérise par un changement de la langue d'enseignement des mathématiques qui passe de l'arabe au français à la transition collège/lycée (14-15 ans).
} 
vide », ce qu'on note $\mathrm{R}(\mathrm{x} ; 0) \neq \emptyset$. Le rapport institutionnel énonce ce qui se fait dans une institution I donnée avec l'objet $\mathrm{O}$, comment cet objet y est mis en jeu. Pour chacun des sujets de I occupant une position $\mathrm{p}$, il exprime qu'il existe un rapport institutionnel à l'objet $\mathrm{O}: \mathrm{RI}$ $(\mathrm{p}, \mathrm{O})$. Ce rapport institutionnel constitue le système essentiel des conditions et des contraintes sous lesquelles se forme et évolue vers un second rapport, ici, le rapport personnel des enseignants à la modélisation algébrique.

\subsection{La notion de praxéologie}

La TAD permet de modéliser toute activité mathématique en termes de praxéologies [11] en la décomposant en un quadruplet (tache, technique, technologie et théorie). Selon ce modèle, les pratiques institutionnelles peuvent être analysées par un découpage en un système de tâches $(\mathrm{t})$ appartenant à des types de tâches $(\mathrm{T})$ [12]. Toute tâche t est accomplie au moyen d'une technique. Chaque technique est justifiée à son tour par une technologie. Celle-ci correspond à un discours rationnel qui permet d'expliquer la technique. Finalement, toute technologie repose elle-même sur les fondements d'une théorie. Dans le cadre de cette étude, cette modélisation permet d'analyser les organisations mathématiques (OM) développées autour de la modélisation dans le domaine algébrique. Nous tentons de relever des aspects plus ou moins ignorés ou cachés dans la dynamique existante entre ces organisations mathématiques (ponctuelles, locales, régionales et globales). Les praxéologies didactiques qui accompagnent la mise en œuvre des organisations mathématiques seront analysées par l'identification de la hiérarchie des niveaux de codétermination didactiques mise en correspondance avec les niveaux de déterminations mathématiques [13] que nous explicitons dans cette étude.

\subsection{Les niveaux de codétermination didactiques}

Une hiérarchie de niveaux de codétermination didactiques [13] permet de préciser l'échelle des organisations praxéologiques, tout en les repositionnant les unes par rapport aux autres. Ces niveaux de codétermination didactique sont divisés en deux niveaux, les niveaux de codétermination supérieurs : ceux de la pédagogie, de l'école, de la société et de la civilisation et des sous-niveaux ou niveaux de codétermination inférieurs, ceux de la discipline, du domaine, du secteur, du thème et du sujet s'étude. Ces sous-niveaux peuvent être mis en rapport avec les niveaux de détermination mathématiques.

-La discipline (NV1) : les mathématiques sont considérées comme un amalgame de domaines d'études.

-Le domaine d'étude (NV2) : l'algèbre correspond à une organisation globale représentant à son tour une amalgamation de plusieurs organisations régionales.

-Le secteur (NV3) fait appel à l'organisation régionale, donc à l'amalgamation d'organisations locales se basant sur la même théorie.

-Les thèmes d'études (NV4) correspondent à l'organisation locale. Ces thèmes sont réunis dans des organisations régionales étiquetées comme secteur d'études.

-Les sujets d'études (NV5) renvoient généralement à un type de tâche T, ce sont des questions et des organisations ponctuelles. Ils se regroupent plus ou moins clairement dans des organisations locales qui représentent les thèmes d'études.

Ces sous-niveaux référent aux différentes divisions qui structurent le curriculum et les organisations mathématiques enseignées selon l'institution considérée. Le tableau suivant illustre cette structuration dans le cas du contexte tunisien. 
Tableau 1. Les niveaux de codétermination didactiques relatifs à la modélisation

\begin{tabular}{|l|l|l|}
\hline Civilisation & Niveau-3 & $\begin{array}{l}\text { Développement professionnel de l'enseignant et } \\
\text { évolution du profil de l'élève. }\end{array}$ \\
\hline Société & Niveau-2 & Approche interdisciplinaire et socio-constructivisme \\
\hline Ecole & Niveau-1 & Cycle secondaire \\
\hline Pédagogie & Niveau 0 & Enseignant tuteur et élève actif \\
\hline Discipline & Niveau 1 & Mathématiques \\
\hline $\begin{array}{l}\text { Domaine } \\
\text { d'études }\end{array}$ & Niveau 2 & Algèbre (praxéologie globale) \\
\hline $\begin{array}{l}\text { Secteurs } \\
\text { d'études }\end{array}$ & Niveau 3 & Modélisation (praxéologies régionales) \\
\hline $\begin{array}{l}\text { Thèmes } \\
\text { d'étude }\end{array}$ & Niveau 4 & $\begin{array}{l}\text { Modélisation numérique, algébrique et fonctionnelle } \\
\text { (praxéologie locale) }\end{array}$ \\
\hline Sujets d'étude & Niveau 5 & $\begin{array}{l}\text { Mise en équations, modélisation par des expressions } \\
\text { algébriques, modélisation par des relations } \\
\text { fonctionnelles. (praxéologies ponctuelles) }\end{array}$ \\
\hline
\end{tabular}

Plusieurs travaux sur la modélisation se placent au niveau thème, ce qui permet de construire des praxéologies mathématiques ponctuelles. La modélisation apparaît ainsi comme un moyen pour introduire un thème ou des notions mathématiques. Une fois, cette introduction réalisée, le système modélisé n'a plus de raison de vivre. D'autres travaux se placent au niveau discipline dans une perspective de développement de compétences multidisciplinaires sans référence directe au contenu mathématique. Cette conception s'accorde avec la notion de curriculum intégré où les contenus mathématiques sont formulés en termes de compétences dont les celles liées à la modélisation. Dans la perspective de la " Realistic Mathematics Education » (RME) [14] on tente plutôt de placer le problème de modélisation au niveau secteur. En effet, l'idée repose sur le fait que les mathématiques sont le fruit d'une activité humaine [15] et l'élève acquiert des connaissances mathématiques formelles en utilisant ses connaissances informelles avec l'aide de l'enseignant. Ces connaissances informelles qui relèvent du concret sont transformées en connaissances formelles via des problèmes contextuels familiers pour les élèves et qui interviennent dans tous les thèmes et les sujets d'étude relatifs à la modélisation.

\subsection{L’approche écologique}

L'approche écologique en didactique [16,17] met en avant en évidence que l'introduction d'un objet dans le système d'enseignement n'est pas naturalisée puisqu'elle elle bouleverse l'équilibre écologique du système en détruisant certaines interrelations entre objets et en construisant de nouvelles. L'étude des conditions écologiques dont les niveaux de détermination didactique [13] permettent de concevoir qu'un objet de savoir ne peut pas vivre de manière isolée mais se trouve en interrelation avec d'autres objets de savoir. Dans cette optique, [16] développe l'idée de la loi du « tout structuré » : la viabilité d'un objet de savoir dépend d'autres objets, sans lesquels il n'a pas de raison d'être. L'analyse écologique d'un objet de savoir s'organise autour de deux notions : l'habitat qui désigne les lieux de vie de l'objet et l'environnement conceptuel de cet objet de savoir et la niche qui désigne la fonction de cet objet dans le système des objets avec lesquels il interagit. Plus précisément, ce sont les outils de l'approche écologique de la didactique qui, à partir d'une analyse du « texte du savoir ", permettent d'identifier le rapport institutionnel à l'objet de savoir en position d'enseignant et de caractériser la place occupée par la modélisation en algèbre. 
« [...] l'analyse écologique du texte d'enseignement [...] permet l'exploration de la clôture institutionnelle de l'univers du savoir enseigné, et consiste en l'analyse des interrelations entre objets, sous-objets et sur-objets présents dans le texte d'enseignement, afin de mettre en lumière, notamment leurs habitats et leurs niches écologiques, les niveaux trophiques, etc. C'est alors à ce niveau que peut être posé le problème de l'identification des objets institutionnels et de l'analyse du contenu des rapports institutionnel et officiel à un objet $\mathrm{O}$ et de leur évolution [...]. » [18]

Ainsi pour apporter des éléments de réponse à ces deux questions : qu'est-ce que le professeur enseigne et comment il l'enseigne? une praxéologie mathématique va permettre de modéliser la réponse à la première question tandis qu'une praxéologie didactique permettra de modéliser la réponse à la deuxième question. Le questionnement écologique permet selon nous, de caractériser les praxéologies didactiques* développées par l'institution à partir de la structuration des niveaux de codétermination didactique mise en avant l'institution. Cela permet ainsi de définir deux rapports institutionnels, l'un en position de professeur, l'autre en position d'élève (topogénèse). » [18] Ils permettent de s'interroger sur l'existence de la modélisation en tant qu'objet d'enseignement. L'articulation des notions d'habitat et de niche avec celles des niveaux de codéterminations didactique permet, selon nous, de décrire de manière plus fine le rapport institutionnel développé à la modélisation algébrique. Ce processus étant souvent conçu comme une modalité pédagogique plutôt qu'un enjeu d'enseignement explicite, nous l'approchons par les niveaux de codétermination auxquels il s'y rattache dans le système éducatif tunisien, en s'interrogeant sur les fonctions remplies par cette activité de résolution des problèmes dans les textes officiels.

Nous empruntons par ailleurs la notion de registre de représentation sémiotique [19] pour caractériser les organisations mathématiques développées dans le manuel autour de la modélisation. Cette démarche consiste en effet à convertir les données du problème posé du registre de départ vers le registre des écritures symboliques (algébriques, numériques ou graphiques). Ces éléments de cadrage théorique étant posés, nous présentons les éléments de référence de notre cadre épistémologique pour cette recherche.

\section{Cadre épistémologique}

\subsection{Le processus de modélisation}

Une revue de littérature sur la modélisation dans l'enseignement des mathématiques nous a permis de dégager certains aspects du processus en vue d'analyser les défis institutionnels auxquels les enseignants sont confrontés pour mettre en œuvre ces pratiques dans leurs classes. Dans la perspective de la RME [14] le processus de modélisation, plus large que celui de mathématisation, s'organise autour de l'activité informelle des élèves. Ces modèles émergents, qui permettent d'avancer dans la résolution de situations « réelles » évoluent, tout au long du processus, par des restructurations apportées par les élèves en rapport avec la situation de départ ou de nouvelles situations. C'est souvent au niveau des pratiques effectives qu'il est possible de construire une " culture de modélisation », à travers le rôle central que l'enseignant est appelé à jouer dans ce processus pour que les élèves puissent mettre en œuvre cette démarche. Ces travaux soulignent les difficultés des enseignants à mettre en œuvre des démarches de modélisation selon une perspective constructiviste où les

\footnotetext{
* Une praxéologie didactique s’organise autour d’un type de tâches, « enseigner une praxéologie mathématique déterminée ». La technique de réalisation de ce type de tâches peut se décrire en utilisant les six types de tâches qui sont autant de moments du processus d'étude de l'organisation mathématique.
} 
élèves sont engagés intellectuellement dans la construction des modèles. Le courant de la RME distingue deux types de mathématisation :

- Une mathématisation horizontale où des outils mathématiques sont mobilisés et utilisés pour structurer et résoudre une situation (du monde réel au monde des représentations, symboles).

- Une mathématisation verticale qui se joue à un niveau purement mathématique (circonscrite au seul monde des symboles) [14]

Le schéma ci-dessous présente les phases de mathématisation horizontale et verticale mise en avant par Barnes [20] pour une situation posée dans un contexte réaliste, le travail de mathématisation horizontale relève de la description de la situation réelle et de la sélection des informations importantes dans la réalité qu'il schématise par « describing ». Le travail de la mathématisation verticale relève pour l'auteur de la traduction des aspects retenus du problème en langage mathématique et du traitement mathématique.

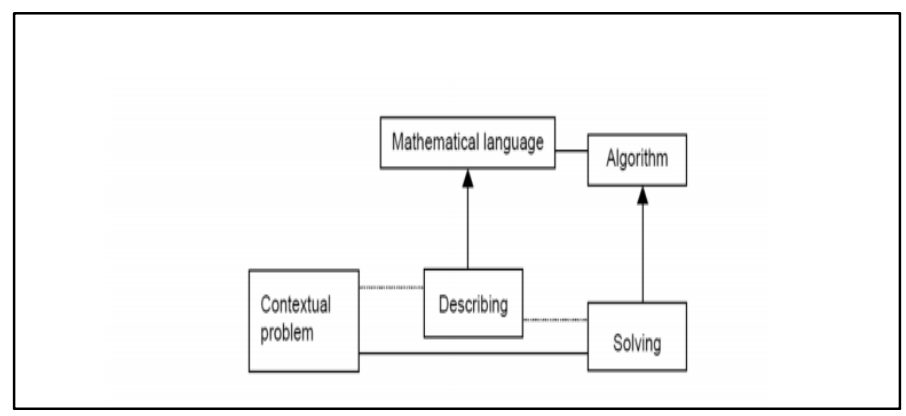

Fig.1. Les mathématisations horizontale et verticale [20].

Blum \& Leiss [21] proposent dans l'enseignement secondaire le cycle de modélisation dont la démarche consiste le plus souvent à appliquer un modèle connu à une situation " réelle », cette situation étant déjà en fait elle-même très fréquemment un modèle réel, obtenu en conférant un habillage pseudo-concret à une situation relevant d'un modèle mathématique bien identifiable.

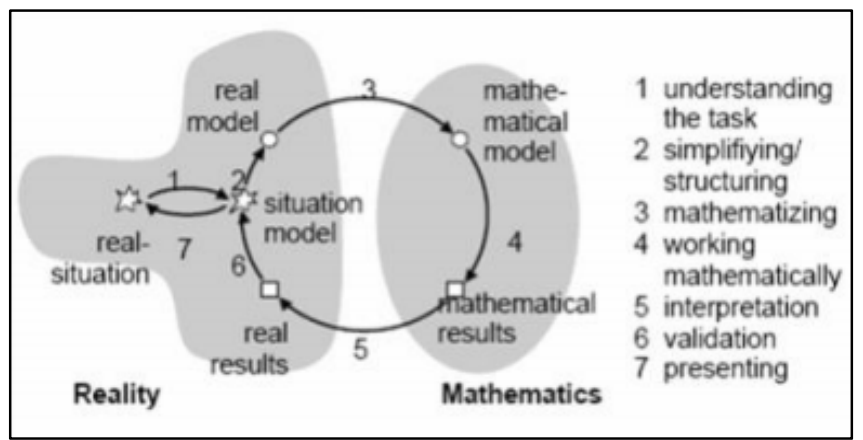

Fig.2. Le cycle de modélisation [21].

Les auteurs en donnent une description en sept étapes :

1. Passage de la situation réelle à la « situation model »-Faire des hypothèses pour le problème et simplifier la situation - Trier les informations pertinentes

2. Passage de la « situation model » au « real model »- Reconnaitre les variables qui influent sur la situation réelle, les nommer, les identifier - Construire des relations entre ces variables 3. Passage du « real model » au modèle mathématique - Mathématiser les variables pertinentes et leurs relations - Simplifier les variables pertinentes et leurs relations si 
nécessaire et en réduire le nombre et leur complexité, Choisir les mathématiques appropriées ainsi que leurs notations pour représenter la situation

4. Travail dans le modèle mathématique - Utiliser des stratégies heuristiques telles que la division du problème en sous problèmes, établir des relations avec des problèmes analogues, reformuler le problème, - Utiliser des connaissances mathématiques pour résoudre le problème

5. Interprétation des résultats mathématiques dans le modèle de situation,

6. Validation ou invalidation de ces résultats dans le modèle de situation (dans le cas d'une invalidation, retour à l'étape 2)

7. Communication des solutions dans la situation réelle.

\subsection{La modélisation algébrique}

En se référant aux travaux cités, nous définissons la modélisation algébrique d'une situation comme un processus qui part de de la situation réelle au modèle algébrique en passant par la « situation modèle » en vue d'un traitement algébrique puis d'une interprétation permettant la validation éventuelle des solutions dans le modèle de la situation. Ainsi, le modèle connu est appliqué à une situation réelle en identifiant les inconnues, variable, paramètre, ... Ces données sont alors symbolisées et les relations entre ces données, qui peuvent aussi bien être des expressions algébriques ou littérales, des équations ou inéquations, ou des relations fonctionnelles (proportionnalités ou autres), sont construites sur la base de compétences à la fois mathématiques et para-mathématiques, convoquant une mathématisation horizontale. L'opérationnalisation de ces relations s'appuie sur des techniques propres à l'algèbre.

Le développement du processus de modélisation algébrique chez les élèves permet d'atteindre plusieurs objectifs didactiques repérés dans des travaux en didactique de l'algèbre. [22,23]

-Sur le plan sémiotique : la mathématisation algébrique offre des possibilités diverses de symbolisation aussi bien des quantités inconnues que des quantités données et permet ainsi le traitement des cas généraux et l'étude des conditions d'existence et d'unicité des solutions par l'introduction de paramètres. Par ailleurs, la démarche de modélisation permet une articulation des registres de représentations sémiotiques, en particulier l'activité cognitive de conversion du registre discursif (dont celui du langage naturel) vers le registre symbolique et le traitement à l'intérieur d'un même registre.

-Sur le plan sémantique : l'analyse en amont et en aval du système visant à sélectionner les informations pertinentes et à contrôler la validité des solutions mathématiques trouvées, implique davantage les élèves dans les tâches qui leur sont confiées et rend ainsi les apprentissages plus motivants.

-Sur le plan praxéologique : La modélisation algébrique permet d'élargir l'environnement technologico-théorique du travail algébrique à travers les opportunités offertes par les tâches de transformation et les techniques de manipulation des expressions algébriques et d'accroître ainsi les connaissances sur le système modélisé et de s'ouvrir ainsi sur de nouvelles questions.

-Sur le plan du rapport au savoir algébrique : le développement de la modélisation algébrique donne du sens aux apprentissages et les rend plus fonctionnels.

Dans le cadre de ce travail, nous cherchons à repérer, dans les pratiques déclarées des enseignants, des indicateurs relatifs aux aspects didactiques évoquées. Les éléments de cadrage théorique et épistémologique étant présentés, nous mettons en avant la méthodologie relative à l'analyse des pratiques institutionnelles autour de la modélisation algébrique et les premiers résultats apportés par cette étude. 


\section{Méthodologie}

Pour analyser les pratiques institutionnelles au début du secondaire nous nous référons aux instructions officielles et au manuel scolaire. Les prescriptions officielles sont analysées à partir des programmes. Pour chaque niveau d'enseignement, nous disposons d'un manuel officiel unique qui constitue la principale référence à suivre par les enseignants. Ces textes officiels traduisent le texte du savoir à enseigner et renseignent sur la perspective curriculaire adoptée par l'institution. Nous croisons des travaux de didactique de l'algèbre relevant des approches sémiotiques et anthropologique. Ces deux approches complémentaires permettent de situer les rapports personnels et institutionnels des enseignants à l'algèbre via la modélisation. Les travaux relevant d'une approche anthropologique proposent des modèles épistémologiques de l'enseignement de l'algèbre. Ils permettent d'interroger les phénomènes didactiques et transpositifs qui déterminent la place et la fonction de l'algèbre à enseigner dans les curriculums. L'approche sémiotique permet de mettre l'accent sur les enjeux sémiotiques de la démarche de modélisation, en particulier les difficultés liées à la conversion entre registres.

Par ailleurs, l'identification des praxéologies mathématiques relatives à la modélisation algébrique s'est inspirée des travaux de Ruiz-Munzon \& al [22] Les auteurs proposent un modèle d'enseignement selon lequel la genèse de l'algèbre s'inscrit dans un processus progressif d'algébrisation des programmes de calcul (pour rappel, l'expression rhétorique d'un programme de calcul est un énoncé du type « choisir un nombre, le multiplier par 4, et ajouter 9 au résultat $»)$. Ce modèle est structuré par :

- Les expressions algébriques. Le type de problème pour motiver la production et l'utilisation des expressions à partir des programmes de calcul est le suivant : deux programmes étant donnés, sont-ils équivalents, i.e. renvoient-ils toujours le même résultat final ? À condition de prendre les « bons » programmes, ce problème met en échec les démarches arithmétiques et par essais/erreurs et nécessite le recours aux expressions.

- Les équations. Le type de problème est le suivant : deux programmes de calcul étant donnés, quelle(s) même(s) valeur(s) entrer dans chaque programme pour qu'ils renvoient le même résultat?

- Les formules algébriques qui font l'objet de la dernière étape du processus

Cette $\mathrm{OM}$ est enrichie pour des raisons liées à notre problématique par la prise en compte de praxéologies institutionnelles. Nous considérons ainsi quatre OM locales de l'OM régionale : modélisation algébrique :

OML1 : La modélisation de situations contextualisées par des expressions numériques.

OML2 : La modélisation de situations contextualisées par des expressions algébriques.

OML3 : La modélisation de situations contextualisées par des équations (ou inéquations).

OML4 : La modélisation de situations contextualisées par des relations fonctionnelles (formules algébriques ou autres relations entre variables)

Ces quatre OM locales sont intimement liées puisque la convocation de certains types de tâches d'une OM locale implique la convocation d'autres types de tâches des OM locales ou des autres. Par exemple, le genre de tâches de OM1 «modéliser le problème par une équation » peut renvoyer à une équivalence de programmes de calcul » par la traduction de chaque programme de calcul, l'un des programmes peut renvoyer à son tour à OM2 au moyen de la mobilisation de formules permettant par la suite la mise en équation.

Nous identifions au sein du domaine algébrique, les niveaux de codétermination didactique inférieurs et les liens qui subsistent. Nous caractérisons ensuite les OML développées autour de la modélisation. Notre étude est exemplifiée par des éléments d'analyse a priori qui mettent en avant les contextes dominants (extra-mathématique, géométrique), les registres de représentation les plus sollicités, les types de tâches suggérés, 
les éléments explicites ou implicites du bloc technologico-théorique relatifs aux techniques installées. Nous confrontons cette analyse à celle de l'étude des pratiques déclarées des enseignants autour de la modélisation par le biais d'un questionnaire. Celui-ci comporte deux parties, la première consiste en des questions semi-ouvertes permettant de recueillir des informations sur la signification accordée par les enseignants à la modélisation en mathématiques et les fonctions qu'ils attribuent à ce processus ainsi que les obstacles éventuellement rencontrés pour mettre en place cette organisation mathématique dans leurs classes. La seconde partie est plus spécifique au domaine algébrique et vise à recueillir des informations sur le rapport des enseignants à la modélisation algébrique, les types de tâches qu'ils choisissent généralement, et comment envisagent-ils de mettre en œuvre cette démarche à partir de ce qui est suggéré dans le manuel officiel (certaines sont d'ailleurs évoquées dans cet article). Notre objectif est d'analyser leurs projets d'enseignement avant la réalisation effective et de caractériser les taches redéfinies par le professeur au regard des tâches prescrites. Les enjeux de la modélisation algébrique via les types de tâches proposées sont-ils toujours visibles pour les enseignants et comment, envisagent-ils de les apprêter? Nous nous limitons dans le cadre de cet article aux résultats relatifs à la première partie du questionnaire, l'analyse de la seconde partie est en cours.

\section{Pratiques institutionnelles}

\subsection{La place de la modélisation en mathématique dans le cycle secondaire}

La réforme de l'enseignement des mathématiques du secondaire mise en place depuis 2004 est particulièrement centrée sur la résolution de problèmes mathématiques et extramathématiques. La prolifération des activités du manuel officiel laisse entrevoir une nouvelle organisation didactique de l'étude qui n'est plus binaire (cours et exercices) et qui renvoie à des pratiques de modélisation simultanément avec la construction de nouveaux objets de savoir algébrique. Cette nouvelle organisation didactique va de pair avec une organisation mathématique développée autour de la résolution de problèmes, par contraste avec les réformes d'enseignement passées où l'aspect structural ou technique domine [5,7]. Les directives des programmes s'organisent autour du développement de compétences disciplinaires et transversales conférant plusieurs fonctions à la pratique de modélisation algébrique.

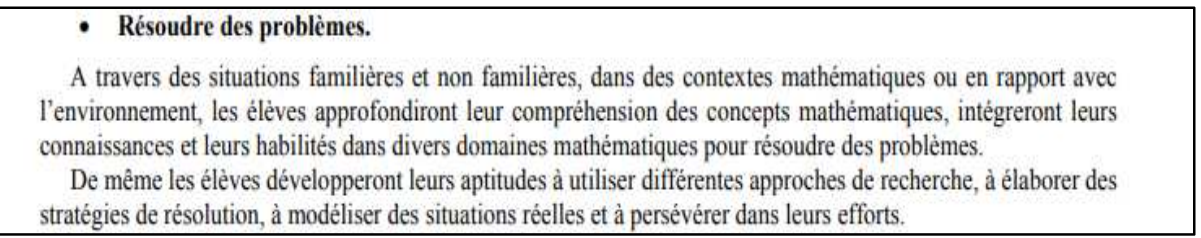

Fig. 3. Extrait des programmes officiels tunisiens du secondaire, 2019.p3.

L'analyse des prescriptions officielles laisse entrevoir plusieurs fonctionnalités attribuées à la modélisation selon que l'on se place du côté des connaissances mathématiques et du raisonnement, ou selon des approches cognitives ou sémiotiques des apprentissages.

-Au niveau des connaissances mathématiques, il s'agit plutôt de développer chez les élèves des compétences dans un domaine des mathématiques ou entre domaines arithmétique/algèbre /géométrie-analyse. 
A travers des activités écrites ou orales, les élèves développeront leurs aptitudes à élaborer une stratégie de calcul (numérique, algébrique, géométrique et statistique) en vue de mobiliser des algorithmes et des procédures.

Fig.4. Extrait des programmes officiels tunisiens du secondaire, 2019.p3.

-Au niveau du raisonnement, il est question de développer l'esprit critique, le raisonnement déductif, l'argumentation et l'abstraction.

\footnotetext{
2. Les élèves développent des raisonnements.

- Ils émettent des conjectures en utilisant un raisonnement inductif, un raisonnement déductif ou un raisonnement par l'absurde ou un raisonnement par récurrence.

- Ils produisent un argument pour valider une affirmation en utilisant des inférences et des déductions.

- Ils développent des chaînes de raisonnement déductif pour prouver une conjecture ou un résultat.

- Ils produisent un contre-exemple pour montrer qu'une assertion est fausse.

- Ils vérifient des résultats et jugent s'ils sont raisonnables.

- Ils distinguent entre une conjecture et un résultat démontré.

- Ils distinguent entre une implication et une équivalence, entre une condition nécessaire et une condition suffisante.
}

Fig.5. Extrait des programmes officiels tunisiens du secondaire, 2019.p4.

-Au niveau de la discipline, cette pratique favoriserait une meilleure compréhension de la vie quotidienne, et permettrait le développement d'une approche interdisciplinaire par la résolution de problèmes concrets ou faisant appel à des domaines d'expérience variés, comme on peut le voir sur cet extrait.

En interagissant avec les autres disciplines et l'environnement, les mathématiques contribuent à leur développement. Elles permettent de comprendre les phénomènes et favorisent les prises de décisions.

Fig.6. Extrait des programmes officiels tunisiens du secondaire, 2019.p3.

-Au niveau cognitif, il s'agit d'un changement conceptuel dans l'enseignement apprentissage des mathématiques qui n'est plus celui des mathématiques modernes abstraites ni celui de la réforme contemporaine à visée calculatoire et technique, mais celui de motiver les apprentissages en cultivant une culture du monde réel.

En interagissant avec les autres disciplines et l'environnement, les mathématiques contribuent à leur développement. Elles permettent de comprendre les phénomènes et favorisent les prises de décisions.

En tant que langue, les mathématiques offrent un moyen de communication précis, rigoureux, concis et universel.

Fig.7. Extrait des programmes officiels tunisiens du secondaire, 2019.p3.

- Au niveau sémiotique, cette approche favoriserait des activités de conversions entre registres qui renforceraient l'aspect cognitif des apprentissages.

Ces différents aspects, déduits de la lecture des programmes, ne sont pas suffisamment explicités et ne sont pas accompagnés d'exemples illustratifs permettant aux enseignants de s'approprier les enjeux de cette approche.

\subsection{La place de la modélisation algébrique dans le programme officiel de 1ère année du secondaire}

Dans le programme officiel de $1^{\text {ère }}$ année du secondaire, la compétence "modéliser»" viserait à « Traduire en langage mathématique une situation réelle (à l'aide d'équations, de fonctions, de configurations géométriques, de graphiques, d'outils statistiques ...). La pratique de modélisation est envisagée comme une méthodologie de résolution de problèmes. 
La mathématisation horizontale n'est pas mise en avant et la transformation d'un problème contextualisé en modèle mathématique est naturalisée en occultant les premières tapes du processus. La mathématisation verticale semble constituer l'enjeu d'enseignement par le développement de techniques permettant d'accomplir les types de taches en jeu dans la résolution de problèmes, comme on peut le voir sur cet extrait du programme.

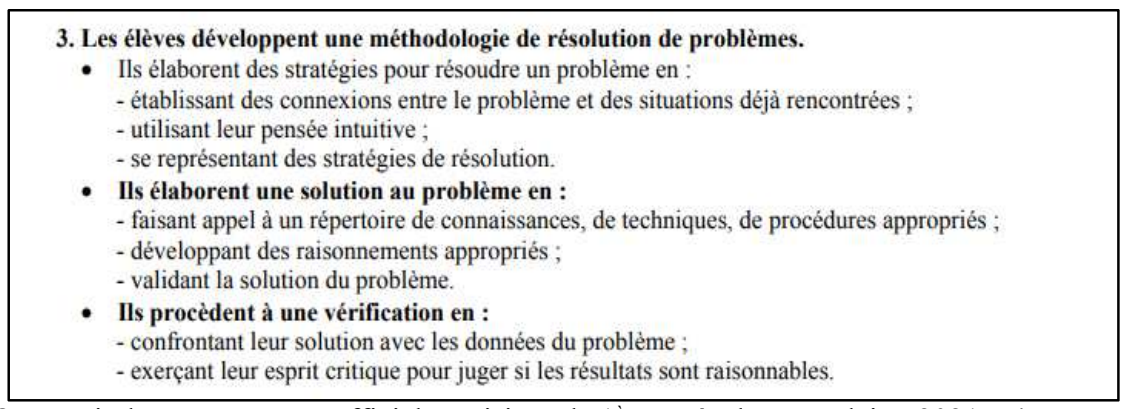

Fig.8. Extrait des programmes officiels tunisiens de $1^{\text {ère }}$ année du secondaire, 2021.p 4.

La modélisation est omniprésente dans les chapitres du programme officiel, « activités numériques », " activités algébriques », " activités géométriques », activités statistiques », " activités dans un repère » et « activités sur les mesures des grandeurs ». Par ailleurs, les programmes sont formulés en termes de compétences que nous considérons d'un point de vue anthropologique comme des éléments du bloc pratico-technique et de savoirs à enseigner qui peuvent être perçus comme des constituants du bloc technologico-théorique. Cela permet de renseigner sur les types de tâches et les techniques attendues. Nous avons procédé à la classification de ces compétences en fonction des secteurs d'activités présents dans le programme et le manuel officiels.

Tableau 2. Compétences exigibles autour de la modélisation en $1^{\text {ère }}$ année du secondaire tunisien.

\begin{tabular}{|c|c|c|c|c|c|c|}
\hline $\begin{array}{l}\text { Secteurs } \\
\text { d'activités }\end{array}$ & $\begin{array}{l}\text { Activité } \\
\mathrm{s} \\
\text { numériq } \\
\text { ues }\end{array}$ & $\begin{array}{l}\text { Activités } \\
\text { algébrique } \\
\mathrm{S}\end{array}$ & $\begin{array}{l}\text { Activités } \\
\text { géométriq } \\
\text { ues }\end{array}$ & $\begin{array}{l}\text { Activités } \\
\text { statistiques }\end{array}$ & $\begin{array}{l}\text { Activités } \\
\text { dans un } \\
\text { repère }\end{array}$ & $\begin{array}{l}\text { Activités } \\
\text { sur les } \\
\text { mesures de } \\
\text { grandeurs }\end{array}$ \\
\hline $\begin{array}{l}\text { Directives } \\
\text { officielles }\end{array}$ & \multicolumn{6}{|c|}{$\begin{array}{l}\text { Résolution de problèmes (PB) dans des situations mathématiques ou en rapport avec } \\
\text { leur l'environnement dans des contextes familiers ou non familiers. }\end{array}$} \\
\hline $\begin{array}{l}\text { Compéten } \\
\text { ces } \\
\text { exigibles }\end{array}$ & $\begin{array}{l}\text { C1: } \\
\text { Modélisatio } \\
\mathrm{n} \text { de } \\
\text { situations } \\
\text { réelles } \\
\text { menant à la } \\
\text { proportionn } \\
\text { alité PB : } \\
\text { taux d'intérêts } \\
\text { simple ou } \\
\text { composé, } \\
\text { les } \\
\text { échelonnem } \\
\text { ents } \\
\text { d'emprunts } \\
\text { ou de prêts, } \\
\text { les remises }\end{array}$ & $\begin{array}{l}\text { C3: } \\
\text { Modélisati } \\
\text { on de } \\
\text { situations } \\
\text { réelles } \\
\text { menant à } \\
\text { des } \\
\text { équations, } \\
\text { des } \\
\text { inéquation } \\
\text { s ou des } \\
\text { fonctions } \\
\text { linéaires } \\
\text { ou affines } \\
\text { C4 : } \\
\text { Résolution } \\
\text { de PB } \\
\text { d'optimisa }\end{array}$ & $\begin{array}{l}\text { C5: } \\
\text { Modélisati } \\
\text { on de } \\
\text { situations } \\
\text { réelles } \\
\text { menant } \\
\text { aux } \\
\text { figures de } \\
\text { base du } \\
\text { plan et de } \\
\text { l'espace } \\
\text { C6: les } \\
\text { élèves } \\
\text { résolvent } \\
\text { des problèmes } \\
\text { d'alignem } \\
\text { ent, de }\end{array}$ & $\begin{array}{l}\text { C7: } \\
\text { Exploitation } \\
\text { des } \\
\text { représentati } \\
\text { ons } \\
\text { graphiques } \\
\text { pour } \\
\text { résumer une } \\
\text { série statistique, } \\
\text { faire des } \\
\text { interprétatio } \\
\text { ns et des } \\
\text { prédictions } \\
\text { sur la } \\
\text { fréquence } \\
\text { d'apparition } \\
\text { de }\end{array}$ & $\begin{array}{l}\text { C9: de } \\
\text { situations } \\
\text { réelles en } \\
\text { produisant } \\
\text { des } \\
\text { représentati } \\
\text { ons } \\
\text { graphiques } \\
\text { C10 : } \\
\text { Analyse et } \\
\text { interprétati } \\
\text { on d'une } \\
\text { représentati } \\
\text { on } \\
\text { graphique } \\
\text { modélisant } \\
\text { une } \\
\text { situation. }\end{array}$ & $\begin{array}{l}\text { C11: } \\
\text { Modélisati } \\
\text { on de } \\
\text { situations } \\
\text { réelles } \\
\text { menant à } \\
\text { des } \\
\text { mesures de } \\
\text { grandeurs } \\
\text { simples ou } \\
\text { composées } \\
\text { - } \\
\text { Intégration } \\
\text { des } \\
\text { connaissan } \\
\text { ces et des } \\
\text { habilités } \\
\text { dans divers }\end{array}$ \\
\hline
\end{tabular}




\begin{tabular}{|c|c|c|c|c|}
\hline $\begin{array}{l}\text { et coûts, } \\
\text { l'évolution } \\
\text { démographi } \\
\text { que } \\
\text { C2: } \\
\text { Résolution } \\
\text { de PB de } \\
\text { dénombrem } \\
\text { ent ou se } \\
\text { rapportant à } \\
\text { des jeux } \\
\text { mathématiq } \\
\text { ues. }\end{array}$ & $\begin{array}{l}\text { tion ou de } \\
\text { point de } \\
\text { rencontre } \\
\text { de deux } \\
\text { mobiles. }\end{array}$ & $\begin{array}{l}\text { concours, } \\
\text { de } \\
\text { constructi } \\
\text { on et de } \\
\text { lieux } \\
\text { géométriq } \\
\text { ues. }\end{array}$ & $\begin{array}{l}\text { phénomène } \\
\text { s aléatoires ; } \\
\text { C8 : } \\
\text { Production } \\
\text { des } \\
\text { représentati } \\
\text { ons } \\
\text { graphiques } \\
\text { de séries } \\
\text { statistiques } \\
\text { ou } \\
\text { chronologiq } \\
\text { ues. }\end{array}$ & $\begin{array}{l}\text { domaines } \\
\text { mathémati } \\
\text { ques pour } \\
\text { mesurer ou } \\
\text { estimer des } \\
\text { grandeurs } \\
\text { simples ou } \\
\text { composées. }\end{array}$ \\
\hline
\end{tabular}

\subsection{La place de la modélisation algébrique dans le manuel officiel de 1ère année du secondaire}

Une analyse des types de taches proposés aux élèves dans leur manuel scolaire ainsi que les techniques attendues nous permis d'identifier les OM locales évoquées autour de la modélisation et le pourcentage associé à chacune. L'analyse quantitative met en avant une importance accordée à l'OML4 qui vise un renforcement des aspects fonctionnels (variable, variation, dépendance fonctionnelle, graphique) via la résolution de problèmes du cadre algébrique.

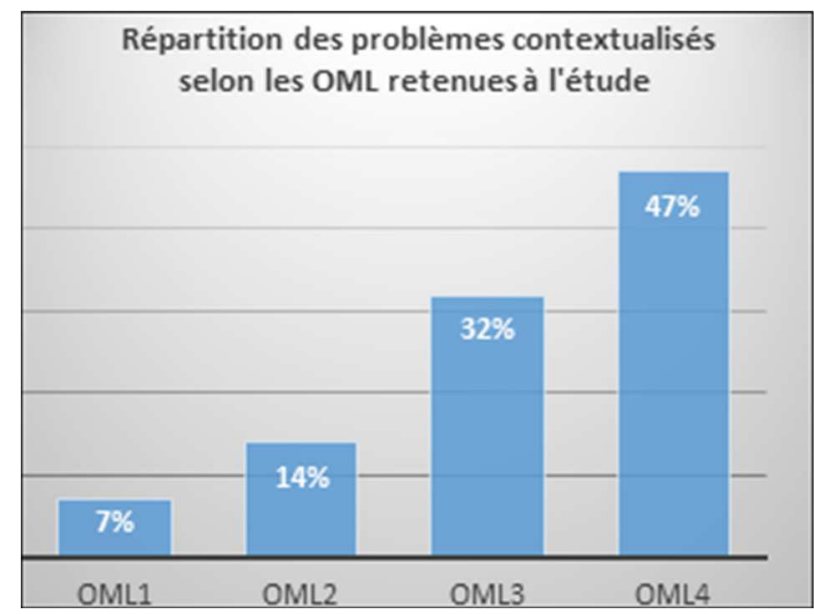

Fig.9. Pourcentages des problèmes selon les organisations mathématiques locales retenues.

\section{Une nouvelle organisation des niveaux de codétermination didactiques inférieurs}

Le registre de l'écriture algébrique est omniprésent et une flexibilité entre les registres graphique et algébrique apparait explicitement à travers les types de tâches proposés. La mise en œuvre de nouveaux outils de représentations accentue la maitrise des situations de proportionnalité. Les notions de fonctions linéaire et affine sont introduites ainsi que les systèmes de deux équations à deux inconnues. Par ailleurs, il apparaît une nouvelle organisation des chapitres qui renvoient à une association des secteurs, équations et fonctions. Ces interrelations entre ces objets de savoir algébriques sont favorisées par la résolution de problèmes contextualisés via les concepts de linéarité, proportionnalité et graphique. La 
modélisation algébrique par un graphique semble jouer un rôle important dans cette nouvelle organisation de l'étude, comme nous pouvons le voir sur cet extrait du manuel.

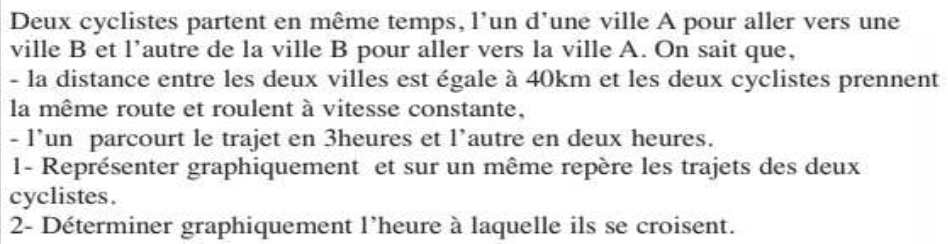

Fig.10. Visualisation du trajet d'un cycliste (République tunisienne, 2019, p. 231).

\section{Les niches et les habitats associés à la modélisation}

L'analyse écologique nous a permis de catégoriser les types de tâches faisant appel à une démarche de modélisation via les problèmes contextualisés proposés dans le manuel officiel en fonction des niches et des habitats associés au regard des niveaux de codétermination didactique mis en avant par notre étude

Tableau3. Les niches et les habitats associés à la modélisation en $1^{\text {ère }}$ année du secondaire tunisien.

\begin{tabular}{|l|l|}
\hline \multicolumn{1}{|c|}{ Habitats } & Niches \\
\hline Arithmético-numérique & $\begin{array}{l}\text { Résolution de problèmes concrets. } \\
\text { Généralisations empiriques } \\
\text { Résolutions de problèmes de proportionnalité }\end{array}$ \\
\hline Algébrique & $\begin{array}{l}\text { Mise en équations } \\
\text { Résolution de problèmes se modélisant par des } \\
\text { inéquations. } \\
\text { Résolution de problèmes simples d'optimisation } \\
\text { Résolution de problèmes menant à des relations } \\
\text { fonctionnelles } \\
\text { Résolution de problèmes se modélisant par des } \\
\text { fonctions linéaires ou affines }\end{array}$ \\
\hline Géométrique & $\begin{array}{l}\text { Résolution de problèmes géométriques } \\
\text { conduisant à des figures de base du plan et de } \\
\text { l'espace. }\end{array}$ \\
\hline Repérage dans le plan/cadre graphique & $\begin{array}{l}\text { Résolution de situations conduisant à un } \\
\text { graphique. } \\
\text { Lecture graphique et interprétation } \\
\text { Résolution graphique et interprétation. }\end{array}$ \\
\hline Mesures de grandeurs & $\begin{array}{l}\text { Résolution de problèmes faisant intervenir des } \\
\text { mesures de grandeurs simples ou composées } \\
\text { Applications de formules }\end{array}$ \\
\hline
\end{tabular}

\section{La place de la modélisation dans la nouvelle organisation de l'étude}

Dans le manuel, trois rubriques mettent en jeu un travail de modélisation selon le statut des problèmes posés dans le manuel officiel, selon qu'il s'agit d'activités introductives, problèmes intégratifs ou des exercices en fin de chapitre. Les activités introductives sont parfois accompagnées de moments d'institutionnalisations de notions ou de procédures et une partie consacrée aux exercices. Nous avons procédé à une analyse de ces situations selon les fonctions qui semblent attribuées à la démarche de modélisation. 
Tableau 4. Les fonctions attribuées à la modélisation selon les caractéristiques des problèmes

\begin{tabular}{|c|c|c|c|}
\hline & $\begin{array}{l}\text { Activités } \\
\text { découverte }\end{array}$ & Problèmes intégratifs & $\begin{array}{l}\text { Exercices } \\
\text { problèmes }\end{array}$ \\
\hline $\begin{array}{l}\text { Fonctions assignées à } \\
\text { la modélisation } \\
\text { mathématique }\end{array}$ & $\begin{array}{ll}\text { Fonction } & \mathrm{de} \\
\text { construction } & \mathrm{de} \\
\text { connaissances } & \end{array}$ & $\begin{array}{l}\text { Fonction } \mathrm{de} \\
\text { réinvestissement et de } \\
\text { formation }\end{array}$ & $\begin{array}{l}\text { Fonction } \\
\text { d'application }\end{array}$ \\
\hline $\begin{array}{l}\text { Caractéristiques des } \\
\text { problèmes }\end{array}$ & $\begin{array}{lll}\text { Découverte } & & \text { de } \\
\text { concepts, } & & \text { de } \\
\text { propriétés ou } & \text { de } \\
\text { procédures } & & \text { de } \\
\text { résolution } & & \end{array}$ & $\begin{array}{l}\text { développer des } \\
\text { compétences } \\
\text { générales } \\
\text { (généralisation, mise } \\
\text { en équations, } \\
\text { manipulation } \\
\text { d'expressions } \\
\text { algébriques }\end{array}$ & $\begin{array}{l}\text { Applications de } \\
\text { formules, de règles, } \\
\text { de procédures, } \\
\text { mobilisation d'un } \\
\text { type de raisonnement }\end{array}$ \\
\hline
\end{tabular}

Dans les activités proposées, le modèle est quasiment initié ou préconstruit et il s'agit pour l'élève d'opérer sur ce modèle en vue de le résoudre. Autrement dit les premières étapes de la mathématisation horizontale sont souvent prises en charge par l'énoncé : l'identification de l'inconnue ou de la variable, la désignation par des lettres et parfois des indications sur les relations algébriques à établir 'équations ou relations fonctionnelles) comme nous pouvons le voir sur ces extraits du manuel.

10 Un cinéma propose à ses clients deux formules.

lère formule : Chaque entrée coûte $2^{\mathrm{D}}, 500$. 2ème formule : Le client paye un abonnement annuel de $50^{\circ}$ et 300 millimes par entrée.

On désigne par $\mathrm{x}$ le nombre d'entrées en une année d'un client.

1- Exprimer à l'aide de $\mathrm{x}$ les dépenses annuelles du client selon les deux formules. 2- A partir de quelle valeur de $\mathrm{x}$, la $2^{\text {bme }}$ formule devient-elle plus avantageuse ?

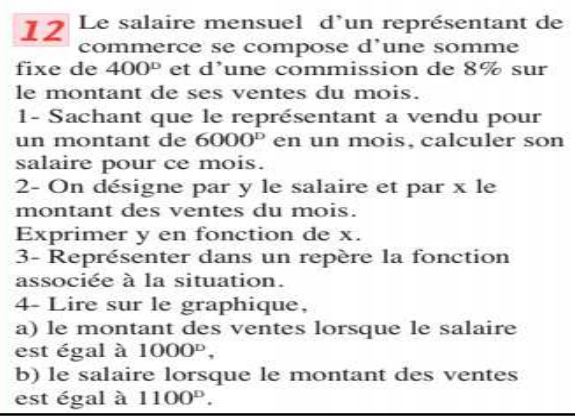

Fig. 11. Problèmes concrets : mise en équation et relations fonctionnelles (République tunisienne, 2019, p.224).

La modélisation algébrique par des formules est également très présente dans les praxéologies mathématiques du type OML2 et OML4. Bien qu'elle semble permettre de dépasser le réductionnisme du registre algébrique par des situations où interviennent simultanément plusieurs variables et plusieurs formes de dépendance, elle semble constituer un enjeu institutionnel important pour dépasser les difficultés éventuellement rencontres par les élèves lors de la modélisation horizontale, comme nous pouvons le voir sur cet extrait. 


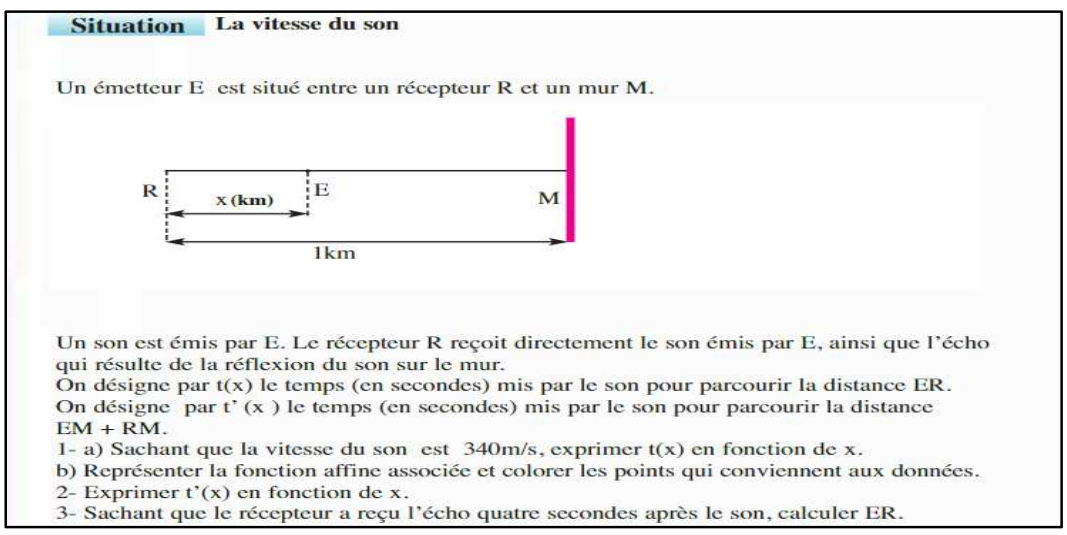

Fig.12. La vitesse du son (République tunisienne, 2019, p.220).

La modélisation algébrico-fonctionnelle qui vise à développer des aspects propres aux fonctions permet aussi de relier implicitement un domaine initial d'existence des grandeurs (le système physique) avec le modèle mathématique (les fonctions mathématiques). Cependant, cette modélisation est souvent prise en charge par l'énoncé, soit par le biais de formules mobilisables par les élèves soit selon un découpage de la tâche en sous tâches donc en organisations ponctuelles fortement guidées. Ce qui semble conférer à ces problèmes un aspect habillage plutôt qu'une occasion de construire un modèle ou d'opérer de manière autonome sur un modèle préconstruit. Même lorsque les situations proposées requièrent une de modélisation horizontale à mettre en œuvre, elles sont accompagnées de «stratégies de résolutions " pour ne laisser à l'élève que l'étape de la modélisation verticale à traiter, comme il apparaît sur cet extrait :

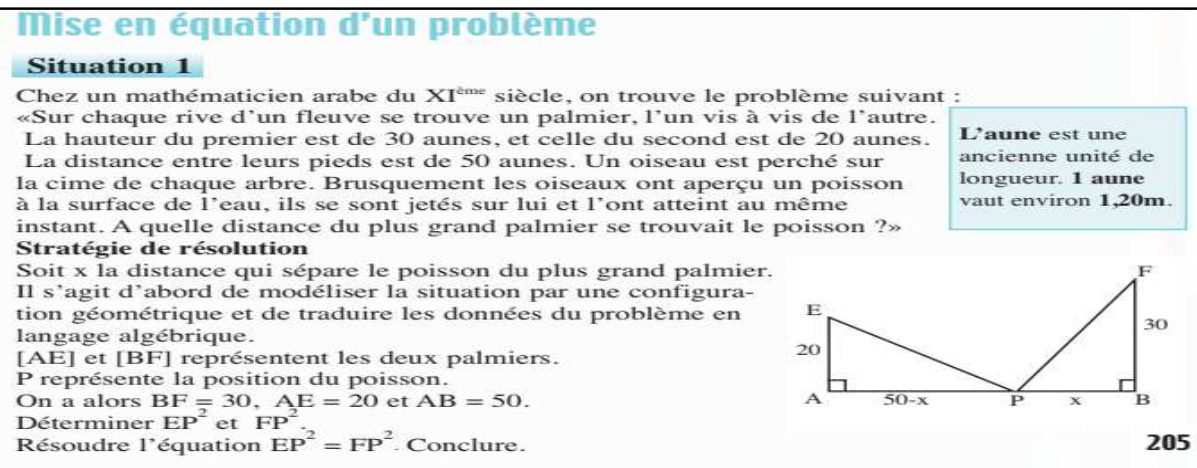

Fig.13. Modélisation d'un problème concret par une configuration géométrique (République tunisienne,2019, p205).

\subsection{Caractérisation des rapports institutionnels développés à la modélisation algébrique : Bilan des analyses}

Les analyses conduites sur le programme et le manuel officiel à la transition au cycle secondaire tunisien mettent en avant des rapports institutionnels aux objets algébriques qui semblent évoluer dans un contexte qui offre des possibilités de disposer d'applications internes et externes aux mathématiques à travers la résolution de problèmes et de situations problèmes. Ces situations mettent en jeu une variété de contextes et une diversification des domaines d'expériences selon une approche à la fois constructiviste et interdisciplinaire des 
apprentissages. Dans cette perspective, une grande place est accordée à la flexibilité entre les registres sémiotiques et les cadres mathématiques. Des dialectiques semblent renforcées entre le registre algébrique et le registre graphique. Ce qui confère à cette approche un aspect plus « dynamique » du savoir algébrique à enseigner par contraste avec l'aspect « statique » qui caractérise les réformes d'enseignement passées. [10] Il semble que, le fait d'installer une pratique de modélisation sur la base d'interrelations entre fonctions et équations permette une meilleure appropriation des concepts en jeu (inconnue/variable ; aspect statique/ variation ; variable/paramètre ; dépendance fonctionnelle) et une entrée dans la pensée fonctionnelle en passant, d'une conception des équations en termes de quantités connues et de quantités inconnues à une conception en termes de variables dépendante et indépendante. Cependant, cette pratique de la modélisation telle qu'elle est conçue dans le manuel qui représente en quelque sorte le texte du savoir à enseigner et la principale référence à suivre par les enseignants nous semble poser des ambiguités qui ne sont pas sans conséquences sur les pratiques d'enseignement-apprentissage de cette démarche. Bien que les programmes officiels se centrent sur la pratique de modélisation, il semble que les situations proposées avec un habillage concret et qui prennent en charge la plupart du travail de mathématisation horizontale ne permettent pas d'atteindre cet objectif. Cet habillage perçu par les enseignants, pourrait expliquer en partie l'impasse faite sur ce genre d'activités qui ne semblent pas valoriser le travail de modélisation ni de développer les compétences requises par cette approche. D'autre part, la prise en charge, dans la plupart des activités, d'une partie importante de la mathématisation horizontale questionne la raison de vivre de ces praxéologies, est ce plus une modalité pédagogique d'enseignement? Renvoie-t-elle à une organisation didactique qui accompagne d'autres organisations mathématiques ? N'est-ce pas paradoxal d'opérer sur des modèles préconstruits alors que les programmes recommandent un développement de compétences dans un perspectif constructiviste?

\section{Pratiques enseignantes}

Les enseignants sont considérés comme les premiers agents des changements et des évolutions au regard des pratiques institutionnelles attendues traduisant le curriculum d'enseignement prescrit. Les pratiques enseignantes qui font l'objet des axes de recherches privilégiés de nos travaux en didactique des mathématiques [5-10] pointent des difficultés d'adaptation aux injonctions institutionnelles. Nous souhaitons approcher ces difficultés du point de vue des pratiques de modélisation, quelles raisons explicatives de l'écart observé entre les pratiques effectives et les pratiques prescrites ? Comment interpréter la stabilité des pratiques observées autour de la modélisation ? Pour tenter d'apporter quelques éléments de réponses à ces questions nous mettons à l'épreuve les hypothèses de recherches suivantes qui guideront notre travail.

Hypothèse 1 : La modélisation horizontale est perçue par les enseignants comme un habillage des problèmes proposés par l'institution qui vient se substituer à une pratique de transmission directe des connaissances mathématiques.

Hypothèse2 : l'absence d'explicitation de la démarche de modélisation horizontale est une contrainte institutionnelle qui pèse sur la sa mise en œuvre effective de cette démarche par les enseignants de mathématiques.

Hypothèse 3 : Les enjeux institutionnels liés aux praxéologies mathématiques développées autour de la modélisation algébrique pour certaines situations du manuel officiel ne sont pas toujours visibles pour les enseignants. 


\subsection{Eléments de méthodologie}

Notre enquête auprès des enseignants est conduite sur la base des pratiques déclarées qui permettent selon nous de dégager certains aspects de notre problématique. Le questionnaire a concerné un échantillon de 57 professeurs de mathématiques du secondaire, exerçant dans différentes régions de la Tunisie. Nous avons choisi de partager ce questionnaire en ligne grâce à l'association tunisienne des sciences mathématiques (ATSM) qui s'est portée volontaire pour diffuser ce questionnaire auprès des enseignants. Certaines questions posées laissent la liberté aux enseignants de donner leurs avis à propos de l'organisation de l'étude et des situations posées dans le manuel officiel. Comment les enseignants interrogés prennent-ils en compte les injonctions institutionnelles au regard de la modélisation en mathématiques ? Quelles contraintes pèsent sur la mise en œuvre de cette démarche dans les classes?

Notre analyse exploratoire est conduite sous l'angle de trois dimensions : la dimension épistémologique en rapport avec la signification attribuée au modèle et à la démarche de modélisation? La dimension fonctionnelle en lien avec le rôle joué par cette démarche dans les organisations mathématiques développées et la dimension organisationnelle en rapport avec les conditions, les contraintes, les défis et les difficultés que ces enseignants associent à l'enseignement et l'apprentissage de cette démarche?

\subsection{Analyse des pratiques déclarées : Point de vue épistémologique}

Cette dimension a été abordée en repérant dans les réponses à la question : Selon vous, en quoi consiste la modélisation en mathématiques ? Qu'est ce qui selon vous caractérise cette démarche ? Les réponses obtenues sont classées dans le tableau qui illustre le pourcentage de chaque type de réponse.

Tableau 5. Les significations attribuées par les enseignants à la modélisation en mathématiques

\begin{tabular}{|l|l|}
\hline Les significations attribuées à la modélisation en mathématiques & $\begin{array}{l}\text { Pourcentage des } \\
\text { réponses }\end{array}$ \\
\hline $\begin{array}{l}\text { Une compétence à traduire une situation donnée en écriture } \\
\text { mathématique. }\end{array}$ & $38 \%$ \\
\hline La capacité à représenter mathématiquement une situation donnée. & $21 \%$ \\
\hline $\begin{array}{l}\text { Une démarche scientifique pour décrire une situation quantitativement } \\
\text { réelle. }\end{array}$ & $7 \%$ \\
\hline Un moyen de faire le lien entre les mathématiques et la réalité & $11 \%$ \\
\hline $\begin{array}{l}\text { La capacité à mobiliser les outils mathématiques nécessaires à la } \\
\text { résolution d'une situation donnée. }\end{array}$ & $20 \%$ \\
\hline Construire un modèle mathématique et le tester. & $3 \%$ \\
\hline
\end{tabular}

Plusieurs réponses s'accordent pour considérer la modélisation en tant que compétence à développer chez les élèves et qui vise à traduire une situation en une écriture mathématique en vue de la résoudre comme l'illustre l'extrait suivant :

Aymen : La compétence " modéliser » viserait à "Traduire en langage mathématique une situation réelle (à l'aide d'équations, des suites, de fonctions, de configurations géométriques, de graphes, de lois de probabilité, d'outils statistiques ...). Utiliser, comprendre, élaborer une simulation numérique ou géométrique prenant appui sur la modélisation et utilisant un logiciel. Valider ou invalider un modèle ». Ce qui caractérise cette démarche est que l'élève est au centre du processus éducatif et celui qui construit son savoir. 
Pour d'autres enseignants dont Amel, il s'agit plutôt d'une conversion du registre du langage naturel vers le registre symbolique en vue de résoudre une situation donnée.

Amel : Une modélisation mathématique c'est la représentation d'un énoncé ou d'un problème mathématique écrit en lettre par un langage purement mathématique faisant appel à des variables en cas de nécessité. Par exemple sous forme d'une équation à une inconnue, d'un système d'équations à deux inconnues et une représentation graphique.

Le même type de réponse est observé chez Mehdi qui considère :

Mehdi : La démarche de modélisation est une démarche scientifique qui consiste à décrire une situation quantitative réelle. Modéliser consiste à écrire en notation mathématique ce qui est exprimé en mots en faisant intervenir des variables au besoin.

Pour d'autres enseignants comme Samar, c'est un moyen de faire le lien entre réalité et mathématiques

Samar : La démarche de modélisation en mathématiques permet aux élèves de résoudre des problèmes concrets et issus de la vie quotidienne. Elle consiste à traduire en langage mathématique une situation réelle. Elle est ainsi un moyen pour faire le lien entre réalité et mathématiques.

La réponse de Fathi est également représentative de plusieurs réponses qui s'accordent pour dire que le plus important est d'opérer sur le modèle mathématique.

Fethi : Traduire en langage mathématique une situation réelle (modélisation) consiste à décrire et comprendre cette situation, mais le plus important ce sont les outils mathématiques pour la résoudre.

Une minorité d'enseignants ont déclaré dans leurs réponses que la modélisation est en réalité un processus à développer sur la base de la construction d'un modèle, la réponse de Sara est typique.

Sara : Il faut faire construire un modèle et tester si ça marche sinon on revient en boucle jusqu'à trouver les solutions au problème.

\subsection{Analyse des pratiques déclarées : point de vue fonctionnel}

La seconde question du questionnaire : Selon vous est-il nécessaire de développer chez les élèves des compétences de modélisation au début du secondaire ? vise à caractériser leur rapport au savoir, leurs conceptions à propos de cette approche du curriculum et les fonctionnalités attribuées à cette démarche. Nous avons classé ces réponses dans le tableau suivant.

Tableau 6. Les rôles attribués par les enseignants à la démarche de modélisation.

\begin{tabular}{|l|l|}
\hline Les rôles attribués à la démarche de modélisation & Pourcentages des réponses \\
\hline Faire le lien entre mathématiques et réalité & $8 \%$ \\
\hline Conduit à élaborer un raisonnement & $13 \%$ \\
\hline Conduit à la construction d'un modèle & $2 \%$ \\
\hline Conduit à opérer sur un modèle préconstruit & $27 \%$ \\
\hline Conduit les élèves à contrôler leurs résultats & $5 \%$ \\
\hline Un habillage du problème & $23 \%$ \\
\hline Pas de rôle spécifique & $22 \%$ \\
\hline
\end{tabular}

Les résultats relatifs à cette première analyse des réponses apportées par les enseignants laissent entrevoir une résistance de certains enseignants à installer cette approche qui selon eux n'a pas vraiment de raison d'être (22\%). D'autres enseignants (50\%) s'accordent pour dire qu'il s'agit plus d'un habillage des problèmes posés ou d'une pratique qui vise à opérer sur un modèle déjà donné, de ce fait, ils ne perçoivent pas l'utilité de la développer en classe 
et préfèrent s'en remettre à des techniques plus calculatoires. Les extraits de Nabil et Aymen illustrent ces points de vue.

Nabil : Pas d'intérêt particulier, cela me semble une perte de temps au lieu de résoudre des équations ou de manipuler avec les élèves des relations fonctionnelles ou des graphiques de fonctions.

Aymen : Je préfère résoudre directement avec les élèves, les équations ou les systèmes d'équations que de perdre du temps à leur faire comprendre le texte de l'énoncé pour rien. Les réponses obtenues laissent entrevoir une conception dominante de la démarche de modélisation comme une modalité pédagogique d'enseignement plutôt qu'un processus de mathématisation qui possède ses propres règles de signifiance et de signifiés, comme l'illustre la réponse de Samia.

Samia : A mon avis c'est indispensable de développer chez les élèves des compétences en rapport avec la modélisation dès le collège et peut être dès le cycle primaire. En effet entraîner un apprenant à résoudre des situations réelles semble être ne nécessite en vue d'apprendre à saisir le lien entre les mathématiques et la vie courante d'une part et entre les mathématiques et les autres disciplines.

\subsection{Analyse des pratiques déclarées : point de vue organisationnel}

Cette dimension des pratiques est abordée sous l'angle des contraintes et des obstacles déclarés par les enseignants, en réponse à la question : A quels genres d'obstacles êtes-vous confrontés pour mettre en place ce type d'approche ? Quelles difficultés particulières rencontrez-vous le plus souvent? Les réponses types apportées par les enseignants à ces questions sont classées dans le tableau suivant.

Tableau 7. Les contraintes évoquées par les enseignants pour mettre en pratique la démarche de modélisation en classe.

\begin{tabular}{|l|c|}
\hline $\begin{array}{l}\text { Les contraintes évoquées par les enseignants pour mettre en pratique la } \\
\text { démarche de la modélisation }\end{array}$ & $\begin{array}{l}\text { Pourcentage des } \\
\text { réponses }\end{array}$ \\
\hline $\begin{array}{l}\text { La surcharge des programmes, l'organisation et la répartition des chapitres } \\
\text { dans les manuels officiels... }\end{array}$ & $22 \%$ \\
\hline Des contraintes de temps & $22 \%$ \\
\hline Le manque de ressources et de formation & $14 \%$ \\
\hline la pratique effective de la modélisation dans le primaire & $20 \%$ \\
\hline $\begin{array}{l}\text { Problèmes langagiers, maitrise insuffisante de la langue française pour } \\
\text { appréhender les situations contextualisées posées au lycée }\end{array}$ & $15 \%$ \\
\hline $\begin{array}{l}\text { Le manque de motivation des élèves et leur réticence à affronter des } \\
\text { problèmes contextualisés }\end{array}$ & \\
\hline
\end{tabular}

Plusieurs réponses (58\%) comme celle de Mourad évoquent des contraintes institutionnelles liées à l'organisation du domaine algébrique, aux contraintes de temps et au manque de ressources pour l'enseignant dans la mise en place de cette démarche en classe :

Mourad : Les difficultés dominantes sont : l'estimation de la durée pour résoudre une tâche de modélisation, le manque de temps pour préparer une tâche de modélisation pour l'enseignant.

Le manque de temps en classe, Ces tâches requièrent beaucoup de ressources supplémentaires.

D'autres enseignants comme Amel remettent en cause le manque de pratiques relatives à cette démarche dans l'enseignement primaire qui leur semble décalé par rapport à ce qui est pourtant attendu par les concepteurs des programmes et des manuels officiels des premiers cycles de l'enseignement de base tunisien. 
Amel :il y a des difficultés en rapport avec le raisonnement par manque de ces types d'exercices dès le cycle primaire et les approches pédagogiques adoptées en classe, il y a une dominance du cours magistral.

Par ailleurs, plusieurs enseignants évoquent les difficultés rencontrées par les élèves pour comprendre les énoncés. Cette difficulté n'est pas propre aux élèves tunisiens mais elle est aggravée par la transition langagière qui accompagne la transition au cycle secondaire $[9,10]$ Naoual : Les compétences relatives à la lecture et l'inférence des textes sont à mon sens importants.

Imed : Le niveau lère année secondaire semble le plus concerné par ces difficultés puisque l'élève trouve des difficultés pour comprendre l'énoncé du problème à cause de passage de l'enseignement de mathématiques en langue arabe en 9ème au langage français en lère année.

Certains enseignants évoquent le manque de motivation des élèves à résoudre des problèmes extra-mathématiques et leur réticence à les affronter contrairement à des problèmes purement mathématiques comme l'illustre la réponse d'Ahlem.

Ahlem : personnellement lorsque je pose des problèmes concrets aux élèves la plupart d'entre-eux abandonnent la tâche ou ne font aucun effort pour les résoudre et attendent que je fasse la correction au tableau, du coup je ne veux plus proposer ce genre de problèmes en classe ni dans les devoirs.

L'analyse exploratoire menée sur la base des pratiques déclarées des enseignants pour le premier volet de cette recherche a permis de mettre en avant certaines caractéristiques des rapports personnels développées par les enseignants autour de la modélisation et ont permis en partie de valider nos hypothèses de recherche. Il semble que les contraintes soulevées par les enseignants sont plus de nature institutionnelles que personnelle. Des contraintes liées à la nature des situations proposées dans le manuel, au temps, à la compréhension des textes, au manque de ressources et d'accompagnement à ces démarches sont autant de facteurs évoqués dans leurs réponses. Ces facteurs constituent un système complexe de contraintes qui peut expliquer en partie la robustesse de certaines pratiques bien qu'expertes à s'engager dans la mise en œuvre de cette démarche. Une majorité d'enseignants est consciente des défis apportés par cette approche innovatrice de l'enseignement des mathématiques. Cependant les points de vue s'accordent à considérer ce processus comme une compétence parmi $\mathrm{d}$ 'autres suggérées par les programmes et qu'il est possible de s'en tenir à l'aspect technique des connaissances mathématiques occultant la mathématisation horizontale du topos de l'élève. Ce qui prime est d'opérer sur un modèle déjà initié ou pris en charge par l'enseignant pour ne laisser aux élèves que l'étape de mathématisation verticale du problème.

\section{Conclusions et perspectives}

Dans cet article, nous nous sommes limités à l'analyse de la première partie du questionnaire qui permet d'expliquer des phénomènes liés entre-autres à la stabilité des pratiques autour de la modélisation. La seconde partie du questionnaire comporte des activités renvoyant aux organisations mathématiques OML3 et OML4 choisies dans le manuel de $1^{\text {ère }}$ année du secondaire et vise à explorer la visibilité, pour les enseignants, des enjeux épistémologiques de ces praxéologies et la manière dont ils envisageraient de les mettre en œuvre leurs classes. Les résultats de cette première analyse montrent en général une compréhension épistémologique limitée de cette approche. Les enseignants du secondaire considèrent souvent les modèles comme des représentations exactes de la réalité. La fonction heuristique (le modèle en tant qu'outil d'exploration des phénomènes) et la fonction prédictive des modèles sont rarement évoquées. Cette étude pointe des aspects de non-conformité des rapports personnels des enseignants aux rapports institutionnels et permet d'approcher la composante cognitive des pratiques au sens la double approche 
ergonomique et didactique [24] qui « résulte de l'analyse de ce que l'enseignant planifie pour agir sur les connaissances mathématiques des étudiants et des étudiantes. Quels savoirs vont être travaillés ? Quels itinéraires cognitifs a-t-il choisis pour les élèves ? » [24]. Ces aspects de non-conformité interrogent la nature des pratiques institutionnelles développées et la visibilité des enjeux liés à la démarche de modélisation pour les enseignants. [25] Cela permet d'interpréter jusqu'à une certaine mesure la logique d'action des pratiques enseignantes et la nécessité pour les formateurs et les concepteurs de programmes de mesurer l'importance de la mathématisation horizontale en accompagnant les enseignants dans cette démarche. Enrichir leurs connaissances via la formation à ce sujet nous parait incontournable. Il nous semble que la mise en place de cette approche dans l'enseignement ne peut se limiter à des prescriptions officielles de façon aussi naturalisée. Des dispositifs collaboratifs par exemple de résolutions de problèmes pourraient contribuer à déstabiliser les pratiques dans le sens attendu par l'institution et motiver les apprentissages dans un perspectif socioconstructiviste. Il nous semble important dans le cadre de la formation initiale et continue des enseignants de développer une « culture de la modélisation » et d'organiser des conditions d'une mise en œuvre adaptée de praxéologies spécifiques à la démarche de modélisation algébrique dans des contextes variés qui seraient plus en conformité avec les rapports institutionnels. Comment construire à long terme un parcours d'étude et de recherche conforme au programme officiel de mathématiques impliquant la modélisation et qui permettrait un développement professionnel dans ce sens ? La formation et les ressources doivent répondre aux priorités exprimées par la politique éducative nationale.

\section{Références}

1. S. Coppé, C. Houdement, Résolution de problèmes à l'école primaire française : perspectives curriculaire et didactique. Actes du 36e colloque de la Commission permanente des IREM pour l'enseignement des mathématiques à l'école élémentaire COPIRELEM. France (2009).

2. M. Artigue, C. Houdement, Problem solving in France: Didactic and curricula perspectives, ZDM, 39, 365-382 (2007).

3. U. Ambrosio, Problem solving: A personal perspective from Brazil, ZDM, 39, 515521 (2007).

4. C. Lajoie, N. Bednarz, Évolution de la résolution de problèmes en enseignement des mathématiques au Québec : un parcours sur cent ans des programmes et documents pédagogiques, CJSMTE, 12, 178-213 (2012).

5. S. Ben Nejma, D'une réforme à ses effets sur les pratiques enseignantes. Une étude de cas : l'enseignement de l'algèbre dans le système scolaire tunisien. Thèse de doctorat. Université Paris VII et Université de Tunis, Tunisie. (2009).

6. S. Ben Nejma, Quel impact d'une évolution du curriculum officiel sur les pratiques enseignantes ? Étude de cas dans le contexte tunisien. Petit x, 82, 5- 30 (2010).

7. S. Ben Nejma, Pratiques enseignantes et changements curriculaires : une étude de cas en algèbre élémentaire. Dans J.-L. Dorier et S. Coutat (dir.), Enseignement des mathématiques et contrat social : enjeux et défis pour le $21 \mathrm{e}$ siècle. Actes du colloque EMF 1133-1142. Université de Genève, Suisse (2012).

8. S. Ben Nejma, Analyse des pratiques enseignantes en rapport avec les praxéologies de mise en équations à l'entrée au lycée : une étude de cas dans le contexte Tunisien. In Abboud, M (éds). Mathématiques en scène, des ponts entre les disciplines. EMF 944-952. Paris, France (2018). 
9. S. Ben Nejma, L'influence d'une perturbation linguistique sur l'activité de modélisation dans le contexte tunisien. RMDM, 4, 3-22 (2019).

10. S. Ben Nejma, L'impact de la langue de formulation d'un énoncé sur les démarches mises en œuvre par des élèves dans une activité de modélisation algébrique. Petit $\mathrm{x}$, 112, 54- 76 (2020).

11. Y. Chevallard, Le passage de l'arithmétique à l'algèbre dans l'enseignement des mathématiques au collège. Petit x, 19, 43-72 (1989).

12. Y. Chevallard. Analyse des pratiques enseignantes et didactique des mathématiques : L'approche anthropologique. Dans Analyse des pratiques enseignantes et didactique des mathématiques : actes de l'université d'été de La Rochelle. ClermontFerrand, France (1998).

13. Y. Chevallard, Organiser l'étude 3. Écologie \& régulation. Dans J.-L. Dorier, M. Artaud, M. Artigue, R. Berthelot et R. Floris (dir), Actes de la XIe école d'été de didactique des mathématiques 41-46 (2002).

14. H. Freudenthal, Revisiting mathematics education. Dordrecht: Kluwer Academic. International Community of Teachers of Mathematical Modelling and Applications. The first twenty-five years (1991).

15. H. Freudenthal, H. Mathematics as an educational task. Dordrecht: Reidel (1973).

16. L. Rajoson, L'analyse écologique des conditions et des contraintes dans l'étude des phénomènes de transposition didactique : trois études de cas. Thèse pour l'obtention du grade de docteur de troisième cycle de l'Université d'Aix-Marseille II (1988).

17. M. Artaud, Introduction à l'approche écologique du didactique - L'écologie des organisations mathématiques et didactiques. Actes de la neuvième École d'été de didactique des mathématiques. Caen, France ,101-139 (1998).

18. M. Artaud, Introduction à l'approche écologique du didactique. L'écologie des organisations mathématiques et didactiques, In M. Bailleul et al. (dir), Actes de l'IXe École d'été de didactique des mathématiques, Houlgate, 101-139 (1997).

19. R. Duval, Registres de représentation sémiotique et fonctionnement cognitif de la pensée. ADSC, 5, 37-65 (1993).

20. H. Barnes, the theory of Realistic Mathematics Education as a theoretical framework for teaching low attainers in mathematics. Pythagoras, 61, $42-57$ (2005)

21. W.Blum, D. Leiss, How the students and teacher deal with mathematical modelling problems? The example "Filling up". ICTMA 12, Chichester, 222-231(2005).

22. N. Ruiz-Munzón, Y. Matheron, M. Bosch, J. Gascón, Autour de l'algèbre : les entiers relatifs et la modélisation algébrico-fonctionnelle. RDM (hors Série), 81-101 (2012).

23. C. Kieran, Learning and teaching algebra in the middle school through college levels: building meaning for symbols and their manipulation. In F. K. Lister (ed.), Second Handbook of Research on Mathematics Teaching and Learning 707-762 (2007).

24. A.Robert, J. Rogalski, Le système complexe et cohérent des pratiques des enseignants de mathématiques : une double approche. CJMSTE, 2, 505-528 (2002)

25. L.Coulange, S. Ben Nejma, C. Constantin, A. Lenfant-Corbin, Des pratiques enseignantes aux apprentissages des élèves en algèbre. RDM (Hors-série) 57-79 (2012). 